\title{
Guest Editorial: Scale Space and Variational Methods
}

\author{
Jan Lellmann ${ }^{1} \cdot$ Jan Modersitzki $^{1}$
}

Accepted: 12 December 2020

(c) The Author(s) 2021

This topical issue of the Journal of Mathematical Imaging and Vision brings together a number of contributions addressing fundamental questions and recent trends in mathematical image processing and analysis: PDE-based imaging and compression, sparsity, gradient flows on manifolds and differential geometry, lifting approaches, computational optimal transport, and fusing existing approaches with elements from machine learning and convolutional neural networks.

Nine contributions have been selected for the topical issue, each advancing the state of the art in the respective field:

Approximating the inverse Radon transform in a stable way is a central object of interest in mathematical imaging. In Computed Tomography Reconstruction with Uncertain View Angles by Iteratively Updated Model Discrepancy, N. A. B. Riis, Y. Dong, and P. C. Hansen show how to introduce a stochastic component for the uncertainties in the model itself. A careful numerical analysis then allows to construct a fast stochastic primal-dual method that achieves a reconstruction quality similar to the known-angles case.

With improving image quality of methods for reconstructing images from sparse data, it becomes tempting to artificially remove data for image compression. In Towards PDE-Based Video Compression with Optimal Masks Prolongated by Optic Flow, M. Breuß, L. Hoeltgen, and G. Radow show how to reduce the cost of the most expensive step, selecting a good sparse subset of data, using motion information from optical flow.

Analysis of PDE-based methods for image filtering is a cornerstone of mathematical image analysis. In PDE Evolutions for M-Smoothers in One, Two, and Three Dimensions, M. Welk and J. Weickert provide a comprehensive analysis for a large class of evolutions based on order- $p$ means. They disprove a long-standing intuition on the mode filter as

Jan Lellmann

jan.lellmann@mic.uni-luebeck.de

Jan Modersitzki

jan.modersitzki@mic.uni-luebeck.de

1 Institute of Mathematics and Image Computing, University of Lübeck and Fraunhofer Institute for Digital Medicine MEVIS, Lübeck, Germany a continuous limit, showing that even in long-standing disciplines of mathematical image analysis there are still new and surprising results to be found.

Studying PDEs based on gradient flows has been a longstanding topic in mathematical image analysis. Recently, an active topic has been flows on manifolds. In Learning Adaptive Regularization for Image Labeling Using Geometric Assignment, R. Hühnerbein, F. Savarino, S. Petra, and C. Schnörr take a new twist on the subject by considering Riemannian gradient flows on the manifold of assignments equipped with the Fisher-Rao metric and integrating them into a supervised framework in order to learn the regularization parameters.

Regularization is also at the core of Block based refitting in l12 sparse regularization by C.-A. Deledalle, N. Papadakis, J. Salmon, and S. Vaiter. They investigate the long-standing issue of contrast loss when using group sparsity-based methods such as total variation and total generalized variation and develop a generalized method for constructing methods to remove this artificial bias.

Computational advancements have made it possible to consider new approaches that rely on embedding images into higher-dimensional spaces before applying PDE-based or variational methods. In Total Variation and Mean Curvature PDEs on the Homogeneous Space of Positions and Orientations, B. M. N. Smets, J. Portegies, E. St-Onge, and R. Duits show how to extend the classical total variation flow and mean curvature flow to this setting, which allows to better recover fine structure and crossings.

Moving into position-orientation space is also the foundation of Cortical-Inspired Wilson-Cowan-Type Equations for Orientation-Dependent Contrast Perception Modelling by M. Bertalmio, L. Calatroni, V. Francheschi, B. Franceschiello, and D. Prandi. They construct a model to correctly predict the perception of optical illusions and-a rare occurence in image processing - prove a negative result, showing that the Wilson-Cowan equation can not be derived as a gradient flow.

Viewing data as elements in a higher-dimensional space of distributions is also at the core of optimal transportbased models, which have seen much interest recently. In 
A Stochastic Multi-layer Algorithm for Semi-Discrete Optimal Transport with Applications to Texture Synthesis and Style Transfer, A. Leclaire and J. Rabin propose an efficient computational method that relies on a hierarchical decomposition. The visual quality that is made possible by these computational advancements indicates strong progress towards better models for the statistics of natural images.

An active topic of research has been to combine mathematical imaging models with convolutional networks and other recent developments from the field of machine learning. In Image Morphing in Deep Feature Spaces: Theory and Applications, A. Effland, E. Kobler, T. Pock, M. Rajković, and M. Rumpf combine the metamorphosis model, which can be viewed as a flow of diffeomorphisms that form a geodesic path in the space of diffeomorphisms, with an image similarity model based on deep features instead of intensity in order to improve the image fidelity.

We thank all authors for submitting their outstanding work to this special issue. We are also deeply indebted to the anonymous reviewers for their very careful, constructive and valuable comments.
Funding Open Access funding enabled and organized by Projekt DEAL.

Open Access This article is licensed under a Creative Commons Attribution 4.0 International License, which permits use, sharing, adaptation, distribution and reproduction in any medium or format, as long as you give appropriate credit to the original author(s) and the source, provide a link to the Creative Commons licence, and indicate if changes were made. The images or other third party material in this article are included in the article's Creative Commons licence, unless indicated otherwise in a credit line to the material. If material is not included in the article's Creative Commons licence and your intended use is not permitted by statutory regulation or exceeds the permitted use, you will need to obtain permission directly from the copyright holder. To view a copy of this licence, visit http://creativecomm ons.org/licenses/by/4.0/.

Publisher's Note Springer Nature remains neutral with regard to jurisdictional claims in published maps and institutional affiliations. 